\title{
Electrochemical Micropyramid Array-based Sensor for in Situ Monitoring of Dopamine Released from Neuroblastoma Cells
}

\author{
Mehmet Senel ${ }^{\mathrm{a} *}$, Muamer Dervisevic ${ }^{\mathrm{b}, \mathrm{c}}$, Sammy Alhassen ${ }^{\mathrm{a}}$, Amal Alachkar ${ }^{\mathrm{a}}$, and Nicolas \\ H. Voelcker ${ }^{\text {b,c,d }}$ \\ ${ }^{a}$ Department of Pharmaceutical Sciences, University of California, Irvine, CA 92697-4625, United \\ States. \\ ${ }^{\mathrm{b}}$ Monash Institute of Pharmaceutical Sciences, Monash University, Parkville, VIC 3052, Australia. \\ ${ }^{\mathrm{c}}$ Commonwealth Scientific and Industrial Research Organization (CSIRO) Manufacturing, Clayton, \\ VIC 3168, Australia. \\ ${ }^{d}$ Victorian Node of the Australian National Fabrication Facility, Melbourne Centre for Nanofabrication \\ (MCN), Clayton, Victoria 3168, Australia.
}

\section{Corresponding Author}

Mehmet Senel

*E-mail: msenel@uci.edu, msenel81@gmail.com

Address: Department of Pharmaceutical Sciences, University of California, Irvine, CA 92697-4625, United States. 


\section{Table of content}

Figure S1. Electrochemical characterization of Au-MPy electrodes using CV and EIS.......S3

Figure S2. CV signal of Au-MPy electrode in the presence of different DA concentrations

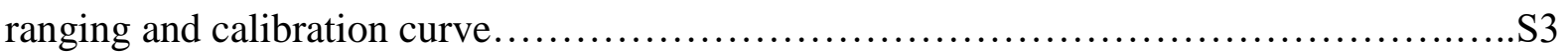

Figure S3. CV signal of DA released from neuroblastoma cells.......................S4

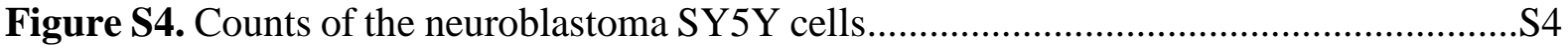



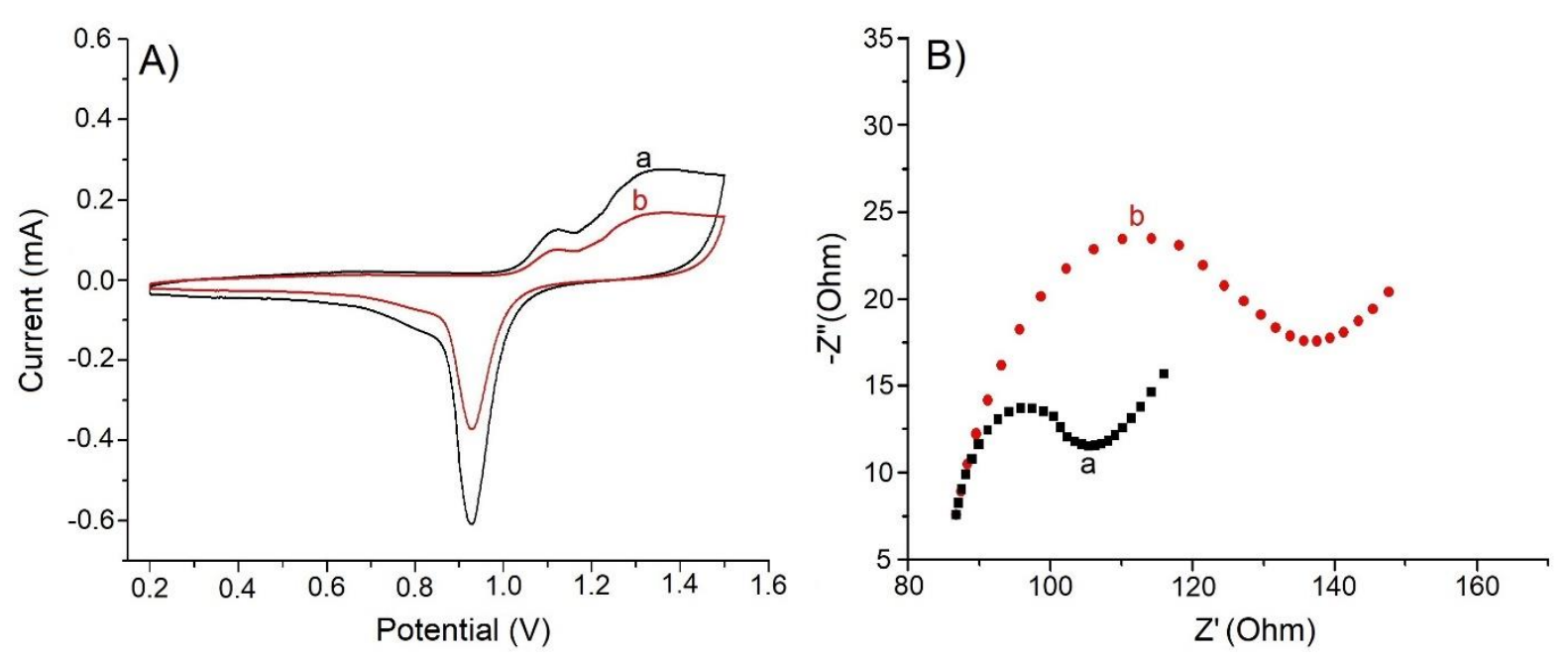

Figure S1. Electrochemical characterization of Au-MPy electrode. A) Cyclic voltammogram plots of (a) Au-MPy and (b) planar Au electrodes recorded in $0.5 \mathrm{M} \mathrm{H}_{2} \mathrm{SO}_{4}$ solution with scan rate of $100 \mathrm{mVs}^{-1}$. B) Electrochemical impedance spectroscopy of (a) Au-MPy and (b) planar Au electrodes recorded in $5 \mathrm{mM}$ of $\mathrm{K}_{4} \mathrm{Fe}(\mathrm{CN})_{6} / \mathrm{K}_{3} \mathrm{Fe}(\mathrm{CN})_{6}(1: 1$ ratio) containing $0.1 \mathrm{M} \mathrm{KCl}$.

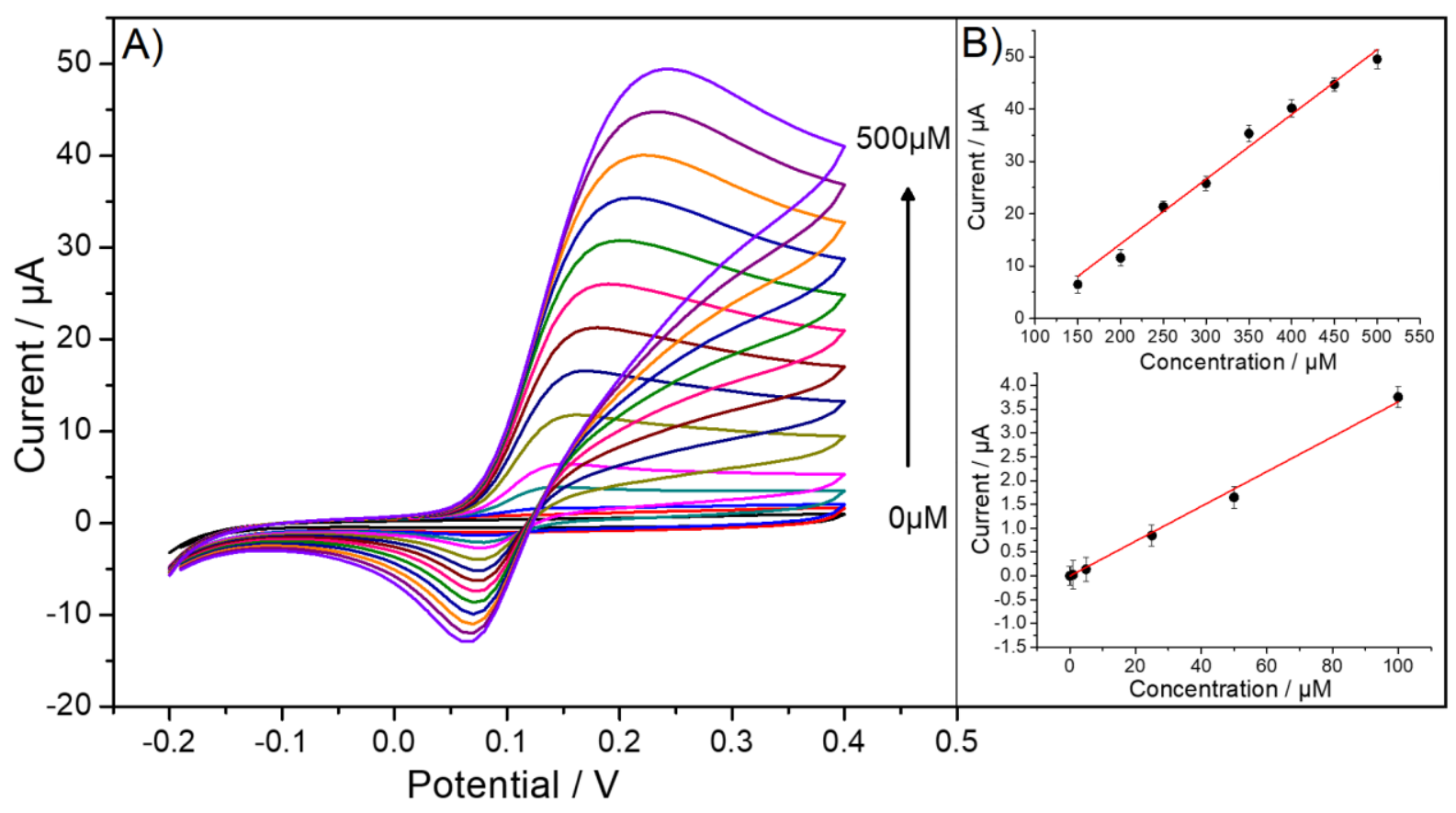

Figure S2. A) Changes in CV signal of Au-MPy electrode in the presence of different DA concentrations ranging from 0.05 to $500 \mu \mathrm{M}$ in DPBS ( $\mathrm{pH} 7.4$ ), and B) calibration curve representing linear correlation between DA and oxidation peak current obtained from the CV signals. 


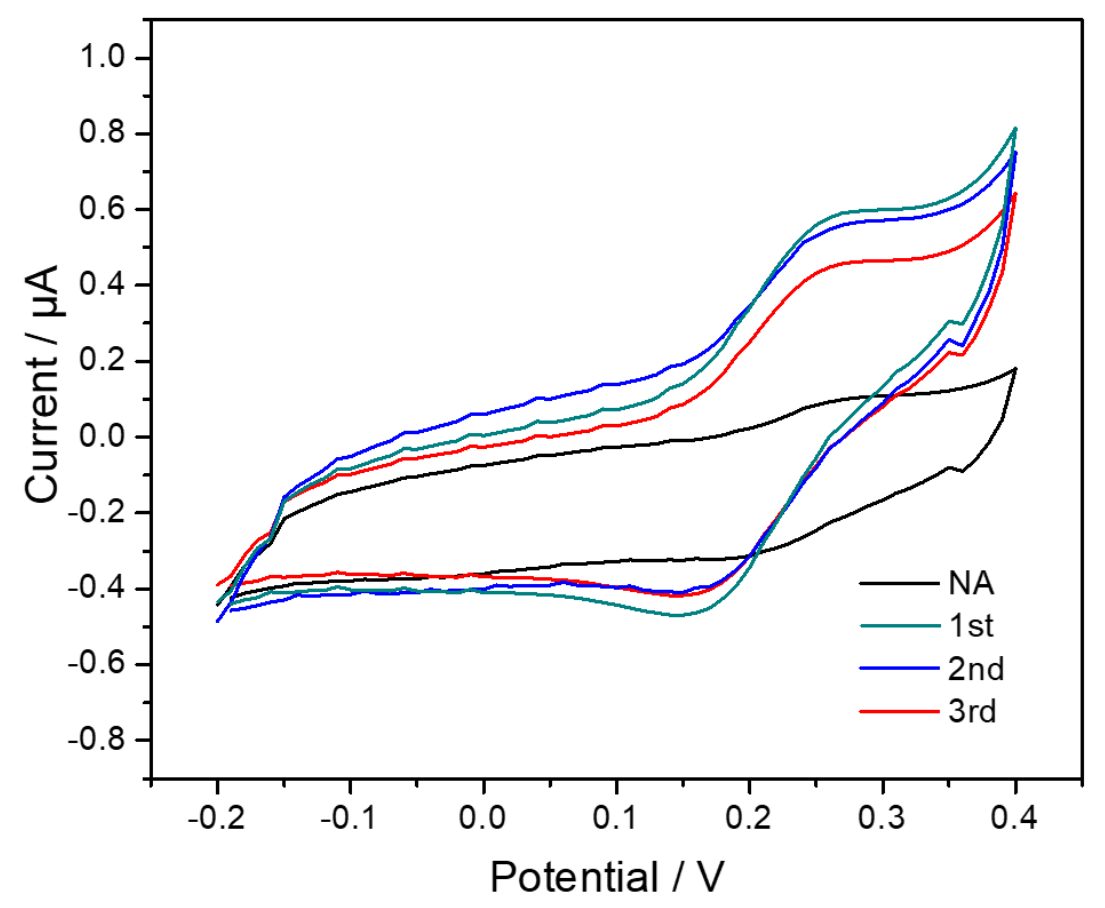

Figure S3. CV signal of DA released from neuroblastoma cells wherein the DA release was stimulated for three times by adding $100 \mathrm{mM} \mathrm{KCl}$.

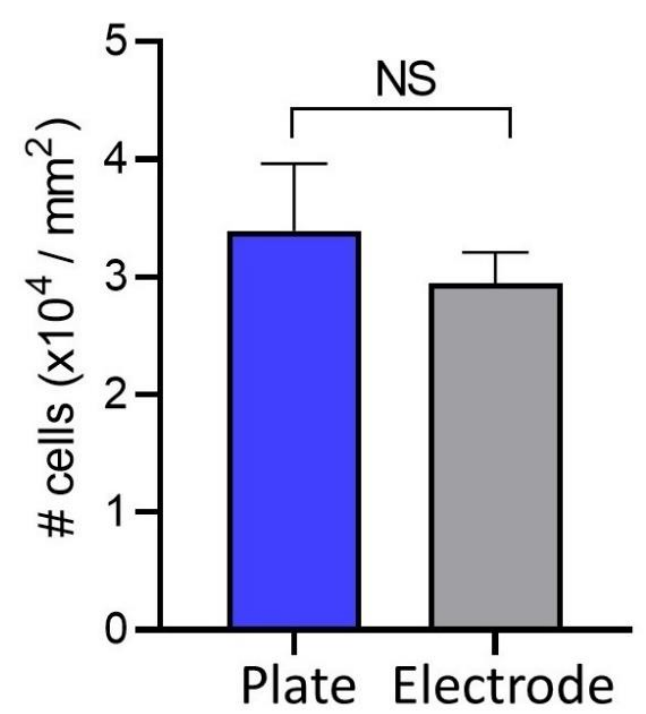

Figure S4. Counts of the neuroblastoma SY5Y cells 24 hours post-culture on $60 \mathrm{~mm}$ culture dish, showing no difference in the number of cells grown on the electrode or on other parts of the plate, $\mathrm{t}$-test, $1=0.71, \mathrm{P}=0.485$ (NS: not significant), $\mathrm{n}=10$ each surface. 